\title{
A INFLUÊNCIA PSICOSSOMÁTICA EM PORTADORES DA SÍNDROME DO INTESTINO IRRITÁVEL
}

\section{PSYCHOSOMATIC INFLUENCE IN PATIENTS WITH IRRITABLE BOWEL SYNDROME}

\author{
Gabriela Machado Duque ${ }^{1}$, lara Salvador Pecemilis ${ }^{1}$, Bárbara Citelis Silva Vargas', Lucas Barcelos Paravidino de \\ Almeida ${ }^{2}$ \\ 1 Acadêmicas de Medicina da Faculdade de Medicina de Campos - FMC \\ 2Pós-Graduando em Psiquiatria - PUC-RJ
}

Autor correspondente: Gabriela Machado Duque; Rua Princesa n 578 Bairro Alphaville; (22)997046600;

gabiduque123@hotmail.com

Faculdade de Medicina de Campos; Av. Alberto Torres, 217 - Centro, Campos dos Goytacazes - RJ, 28035-581

\section{RESUMO}

O propósito dessa revisão de literatura é promover uma fonte de dados que fundamente uma relação entre a síndrome do intestino irritável e desordens psiquiátricas, como a depressão e ansiedade. A síndrome do intestino irritável faz parte de um grupo de patologias com influência do eixo cérebro-intestino a partir de suas sinapses e neurotransmissores, sendo atribuída como um distúrbio na função gastrointestinal (DFGI). Com essa síndrome cada vez mais incidente na sociedade moderna, se torna imperativa a necessidade de aperfeiçoamento das técnicas diagnósticas, levando em consideração a importância de uma equipe multidisciplinar. Com essa abordagem, compreende-se um acréscimo tanto no diagnóstico quanto na relação médico-paciente.

Palavras-chave: síndrome do intestino irritável, transtornos psiquiátricos, psicoterapia e transtornos psicossomáticos.

\begin{abstract}
This literature review's aim is to provide a data source supporting a relationship between irritable bowel syndrome (IBS) and psychiatric disorders, such as depression and anxiety. IBS is part of a pathology group influenced by brain-intestine axis from its synapses and neurotransmitters, being classified as a functional gastrointestinal disorder (FGID). With this syndrome's presence growing in modern society, it becomes imperative to upgrade diagnostic techniques, taking into account the importance of a multidisciplinary team. Both the diagnosis and the doctor-patient relationship are improved by this approach.

Keywords: irritable bowel syndrome, psychiatric disorders, psychotherapy and psychosomatic disorders.
\end{abstract}




\section{Objetivos}

Revisar dados sobre desordens psiquiátricas e sua relação com a SII. Determinando assim, informações atualizadas e baseadas no contexto atual do status psiquiátrico da sociedade, através da coleta de dados sobre a DFGI direcionados aos especialistas da saúde mental e demais especialidades envolvidas.

\section{Métodos}

Foram pesquisados diferentes estudos com assuntos substancialmente semelhantes nos principais bancos de dados eletrônicos (PubMed, MEDLINE, Cochrane library, Scielo e UpToDate). Incluídas publicações do ano de 2003 a 2020 que obtivessem palavras-chave "Irritable Bowel Syndrome", "Functional Bowel Disorders", "Depression" "Anxiety" e "psychosomatic". Em seguida, direcionamos a obtenção de dados a partir dos critérios selecionados.

\section{Introdução}

A síndrome do intestino irritável (SII) faz parte dos distúrbios funcionais gastrointestinais (DFGI) e, algumas evidências sugerem que fatores psicológicos possuem um papel importante em sua patogênese (Surdea-Blaga et al., 2016). Seu acometimento tem prevalência global de $11 \%$ (Canavan et al., 2014), variando entre 10 e 25\% (Lovell e Ford, 2012), em amostras da comunidade, o que gera gastos consideravelmente altos para o sistema de saúde e impactam na qualidade de vida do paciente, no funcionamento social e acarreta em afastamento do trabalho (Spiller et al. 2007). De forma sindrômica, pode-se observar dor abdominal, a qual possui intensidade variável, e as alterações do hábito intestinal, podendo adquirir um subtipo com predomínio de constipação (25\%) ou de diarreia (25\%), mista (25\%) e até mesmo não classificada (Lacy et al., 2016).

Segundo Farzaneh et al. (2012) existem dados epidemiológicos que consolidam a relação da SII associada a alguns fatores, como baixa qualidade de vida e função social prejudicada. Além disso, foram salientadas disfunções psicossociais e observado que $50 \%$ a $60 \%$ dos pacientes com SII apresentam sintomas depressivos, fazendo uma alusão entre SII e status psiquiátrico (HausteinerWiehle e Henningsen, 2014). Evidências crescentes mostram que, em comparação com indivíduos saudáveis, os portadores de condições psiquiátricas e SIl apresentam diferenças em estudos de imagem cerebral, o que leva a crer em uma associação entre ambas (Labus et al., 2014; Tillish et al., 2011; Aizawa et al., 2012; Coss-Adame et al., 2014).

Outro estudo demonstrou que as vias nervosas aferentes, bem como sinais humorais transmitem informações do trato gastrointestinal ao sistema nervoso central. A informação é processada em várias áreas do cérebro e frequentemente motivam feedbacks no intestino (Hetterich e Stengel, 2020). Possíveis desregulações no eixo cérebrointestino, podem acarretar em alterações intestinais quanto a sua motilidade, reação imune ou permeabilidade conduzindo às respostas inflamatórias que contribuem para a hipersensibilidade visceral (Soares, 2014). Essa descoberta de interações e sinapses entre o sistema nervoso central e o intestino, reflete nos critérios de Roma IV, onde a SIl se encontra no grupo de doenças em interações desarmônicas do eixo anteriormente citado (Drossman et al., 2016). Essa suposta interação entre tais órgãos, reforça a relação de doenças psiquiátricas com a respectiva disfunção gastrointestinal e consequentemente nas possíveis opções de tratamento. Quanto às desordens psiquiátricas frequentes, enaltecem os transtornos de ansiedade (30-50\%), depressão (70\%), e, menos frequentemente, distúrbios alimentares (Creed et al.,2005).

\section{Somatização na SII}

Levando em consideração a característica psicossomática envolvida na SII, vale reconhecer as influências que o modelo biopsicossocial abrange. $O$ modelo de Liu et al. (2017) considera fatores biológicos e psicossociais como condições predisponentes, tais como genética, hereditariedade e traumas, principalmente relacionados à infância e a uma maior.

No início da vida o eixo hipotálamo-hipófiseadrenal (HPA) possui um período hiporresponsivo ao estresse, que se traduz por níveis menores de tensão. Nesse período há uma hipótese que se baseia em proteger o desenvolvimento cerebral da produção aumentada de glicocorticóides. Todavia, no eixo HPA podem decorrer alterações epigenéticas produzidas em razão dos episódios traumáticos na infância, sucedendo em um feedback negativo dos glicocorticóides e gerando uma maior predisposição 
à distúrbios psicossomáticos em adultos (O ${ }^{` M a h o n y ~}$ et al., 2019).

O estágio primitivo pré e pós nascimento é essencial para o avanço das vias nociceptivas e se traduz em uma enorme plasticidade. Os modos pelos quais os eventos traumáticos precoces interferem na hipersensibilidade visceral ainda precisam ser determinados, porém é notório que essas alterações epigenéticas interferem em sua patogênese (Liu et al., 2017). Outros fatores que poderiam precipitar a DFGI em questão seriam a falta de apoio social, eventos estressantes e infecção intestinal.

Quanto às influências da microflora fecal, e sua ecologia complexa e ainda pouco compreendida, existem estudos que determinam que mudanças em sua composição poderiam estar associadas a doenças como a síndrome do intestino irritável. Alguns dados sugerem que tal microbiota e m indivíduos com sıl se mostra constitucionalmente diferente quando comparados com grupos saudáveis e varia de acordo com determinada predominância de sintomas envolvidas na patologia (Kassinen et al., 2007; Malinen et al., 2005; Rajilić-Stojanović et al., 2011; Jeffery et al., 2012). Por outro lado, alguns levantam a hipótese de a composição da microflora seja capaz de modular as vias sensoriais viscerais no início da vida a partir de eventos traumáticos precoces, desequilibrando tal constituição e executando um papel importante decorrente de tal modulação condutoras de impulsos nervosos (Liu et al., 2017).

$\mathrm{Na}$ obtenção de dados a respeito da influência psicológica no surgimento da SII, desordens como ansiedade, depressão e percepções negativas foram contribuintes para a origem e prognóstico dos sintomas ao longo do tempo (Deary et al. 2007; Hauser et al. 2014). Uma revisão sistemática com meta-análise demonstrou um aumento considerável da SII em pacientes que apresentavam depressão e ansiedade quando comparado com o grupo controle sem DFGI, incluindo as análises de todos os subtipos da SII (Lee et al., 2017). Um argumento para tais achados seria uma alteração no sistema de liberação intestinal de serotonina (5-hidroxitriptamina, 5-HT), onde níveis elevados de 5 -HT poderiam favorecer a diarreia através do receptor $5-\mathrm{HT}$, e o aumento da liberação pós-prandial de 5-HT teria relação fisiológica em pacientes portadores da SII com predomínio de diarreia (SII-D) (Spiller, 2008). Quanto ao subtipo da SII com predomínio de constipação (SII-C), um suposto desequilíbrio da secreção de 5 -HT estaria presente, e os níveis elevados de depressão e ansiedade neste subtipo explicariam a baixa responsividade desse neurotransmissor nas regiões central e periférica investigadas (Muscatello et al., 2010). Como resultado, todos os subtipos apresentaram forte inerência com desordens como a depressão e ansiedade tendencialmente agravadas (Lee et al., 2017).

Segundo O'Mahony et al. (2017), foi observado que portadores da SII possuem alterações em suas sinalizações cérebro-intestinais, assim como os pacientes que possuem transtornos psiquiátricos reforçando a possibilidade da associação entre essas patologias. Um estudo demonstrou duas premissas diferentes decorrentes dessa possível comunicação. Uma delas seria que os sintomas abdominais secundariamente influenciam a intercorrência de ansiedade e depressão, a outra seria que esses transtornos psiquiátricos, assim como outros fatores psicológicos, como o estresse, seriam responsáveis por induzir às respostas sensoriais e motoras no trato gastrointestinal através da aferência vagal e simpática (Baneriee et al., 2017). Além disso, portadores de SII que não possuem doença psiquiátrica confirmada poderiam, ainda sim, apresentar sintomas psicossomáticos (Surdea-Blaga et al., 2016) se beneficiando da avaliação e possível interferência de pluraridade profissional, como psicólogos e psiquiatras.

\section{Diagnóstico}

A confirmação da SIl se dá através da clínica do paciente, utilizando-se de métodos que auxiliam na exclusão de outras patologias, como os testes laboratoriais (hemograma, calprotectina fecal, proteína $C$ reativa, exame parasitológico de fezes, marcadores sorológicos P-ANCA e ASCA) e exames de imagem, como a colonoscopia e ultrassonografia de abdômen para afastar ou confirmar possíveis diagnósticos, se atentando a sintomas variados e alarmantes como perda de peso inexplicável e sangramento retal não elucidado. De acordo a necessidade e com a investigação, o especialista se orienta, podendo aderir de outros métodos para fundamentar seu diagnóstico.

Os critérios de Roma IV, difusamente utilizado pelos especialistas, definem essa patologia 
como uma desordem envolvendo funções cérebrointestinais. Esses critérios se baseiam na presença de dor abdominal recorrente associada a pelo menos 2 dos seguintes sintomas: (1) relacionada a evacuação (melhora ou piora), (2) alteração na frequência das evacuações (aumento ou diminuição do número) e/ou (3) no aspecto das fezes (definido pela escala de Bristol) (Longstreth et al., 2006). No entanto, apesar da notoriedade de um bom diagnóstico clínico dado por um gastroenterologista, também se torna primordial a coadjuvância com o uso do modelo biopsicossocial na investigação (Tanaka et al., 2011). Portanto, elucida-se a necessidade de uma equipe multidisciplinar, envolvendo especialistas da saúde mental na participação da conduta do paciente. Para ratificar essa necessidade, buscamos apontamentos que demonstram a média de que $40 \%$ dos indivíduos que atendem aos critérios necessários para a SII, não possuem um diagnóstico formal confirmado, exigindo assim, um novo olhar para tal patologia (Sayuk et al., 2017).

\section{Tratamento}

A conduta terapêutica deve ser introduzida no exato momento do diagnóstico, quando o médico explica ao paciente sua condição patológica crônica, fornecendo informações sobre sua situação (Lacy et al., 2016). A terapia inicial se baseia a partir de uma relação de confiança e segurança entre o médico e o paciente, além da compreensão necessária à continuidade do tratamento. Levando em consideração a integralidade e singularidade de cada indivíduo, nos casos de sintomas que não comprometem a qualidade de vida do portador da SII, o recomendado inicialmente são alterações do estilo de vida e dieta, excluindo primariamente o uso de fármacos. De acordo com Wald (2020) nos sintomas leves a graves sem resposta às tentativas iniciais, onde há prejuízo da qualidade de vida, preconiza-se terapia farmacológica como tratamento.

\section{Tratamento não-farmacológico Dieta e exercício físico}

É fundamental ajustes quanto à dieta, tendo como ponto de partida alguns dados que estipulam que cada paciente possui certa intolerância própria, levantando a necessidade do autoconhecimento e da história dietética cuidadosa com erradicação de tal consumo. Existem evidências que pacientes com a DFGI em questão, se beneficiam da exclusão de alimentos com produção de gás, como por exemplo, lactose e glúten. Foi analisado e confirmado que o consumo de carboidratos e polióis de cadeia curta facilmente absorvíveis e fermentáveis (FODMAPs) aumenta velocidade da fermentação bacteriana (Halmos et al., 2014). O estresse também se correlacionou com a dieta e a microbiota intestinal e o papel dos metabólitos produzidos por ela, refletindo em alteração da função intestinal e diversos processos regulatórios no eixo cérebrointestino (Tana et al., 2010).

A atividade física também mostrou um benefício potencial em relação aos sintomas da SII, além das vantagens gerais do exercício físico para a saúde. Em um estudo clínico randomizado, 102 pacientes com SII foram orientados para aumentar a atividade física ou manter os níveis atuais de esforço, sendo que 75 foram até o final do estudo (38 no grupo praticantes de atividade física e 37 no grupo controle). Após 12 semanas, observou-se uma tendência de mais pacientes praticantes de atividade física mostrando melhora clínica na gravidade dos sintomas da SII em comparação com o grupo controle ( 43 versus $26 \%, p=0,07$ ). Além disso, os pacientes no grupo de atividade física tiveram um prognóstico significantemente melhor da SII em comparação aos controles (8 versus $23 \%$ ) (Johannesson et al., 2011).

\section{Psicoterapias}

Vale ressaltar que as diretrizes sugerem a inclusão das terapias alternativas além do tratamento farmacológico (Ford et al., 2008), de modo a estabelecer uma abordagem multifatorial e eficiente, permitindo a participação de equipe variada voltada em uma mesma finalidade, o bemestar do paciente. Um método que se mostrou benéfico no tratamento não farmacológico, foram as terapias com abordagem psicológica, as quais se mostram como um diferencial na evolução dos pacientes, principalmente naqueles que não obtiveram melhora através dos tratamentos convencionais (Hetterich e Stengel, 2020). A psicopatogênese da doença traduz a necessidade de avaliação individual, analisando possíveis desordens psiquiátricas e permanece como grande recurso terapêutico, mesmo nos pacientes que não se encaixem nos critérios de ansiedade e depressão 
(Baneriee et al., 2017). O propósito da terapia psicológica visa compreender o paciente, sua disfunção, os aspectos psicossomáticos e os possíveis transtornos psiquiátricos intrínsecos a ela. Dessa maneira, possui como objetivo principal a atenuação psicossomática, visto o potencial desencadeador e/ou exacerbante na SII. Os principais tipos de psicoterapia são: hipnoterapia, terapia cognitivo-comportamental, psicoterapia dinâmica e psicoeducação. Em um estudo realizado, apontou-se que a psicoterapia pode promover uma melhora dos sintomas em aproximadamente $25 \%$ dos portadores de SII, sendo estatisticamente um percentual relevante (Ford et al., 2008).

Flik et al. (2019), através de um ensaio clínico multicêntrico randomizado controlado, demonstrou que a hipnoterapia pode ser considerada uma opção de tratamento para todos os portadores, independente da severidade ou do subtipo.

Outra possível técnica seria a terapia cognitiva comportamental (TCC) que, de maneira geral, é baseada na redução de medos irracionais e controle do comportamento dos indivíduos (Hetterich e Stengel, 2020). Sua eficácia se manifesta através da redução do estresse psicológico, o que resulta em melhora dos sintomas relacionados ao trato gastrointestinal, como por exemplo, da hipersensibilidade visceral (Surdea-Blaga et al., 2016).

Uma meta-análise inferiu que a TCC foi eficiente na redução dos sintomas, melhora da qualidade de vida e estado psicológico, sendo considerada uma medida promissora na avaliação pós-tratamento (Li et al., 2014).

A psicoterapia dinâmica é recomendada pela Organização Mundial de Gastroenterologia no tratamento da SII (Quigley et al., 2016) e tem seu viés focado nos conflitos intra e interpessoais, e como essas relações contribuem para o aparecimento e manutenção dos sintomas (Hetterich e Stengel, 2020).

Outro tipo de intervenção é a psicoeducação, configurando-se em sessões educacionais com o objetivo de levar ao paciente informações sobre sua doença, causas, tratamentos entre outros aspectos (Shah et al., 2020), o que se mostra interessante, visto que é uma patologia crônica. Considerada uma medida excelente permitindo estabelecer uma relação médico- paciente consistente, e impactando positivamente no curso da doença e na manutenção dos sintomas (Hetterich e Stengel, 2020). Um estudo clínico randomizado avaliando o método da psicoeducação inferiu que os pacientes no grupo intervenção obtiveram uma melhora significativa nos sintomas gastrointestinais, na hipersensibilidade visceral, no quadro da depressão e na qualidade de vida, quando comparados ao grupo controle (Labus et al., 2013).

As terapias psicológicas agem de forma diferente do tratamento farmacológico, isso porque atuam em áreas específicas do cérebro, modificando respostas cognitivas, comportamentais e emocionais que estão relacionadas ao eixo cérebrointestino. Dessa maneira, contribuem para a redução dos aspectos psicossomáticos e, consequentemente nas manifestações clínicas da doença (Ford et al., 2019).

\section{Tratamento farmacológico}

Em outro contexto, e levando em conta a individualidade de cada paciente, não se pode dispensar em grande parte dos casos o tratamento farmacológico da SII, que se embasa no controle da sintomatologia moderada a grave dos portadores. Seu objetivo principal se encontra na promoção da qualidade de vida ao paciente em quadros incapacitantes ou prejudiciais, e que não obtiveram resultados com as condutas iniciais. A predileção para o tratamento se respalda na sintomatologia e no subtipo predominante. Como considerado anteriormente, costuma-se usar a intervenção farmacológica no controle de crises (como exemplo, diarreicas), mas também se dispõe de terapia farmacológica contínua (por exemplo, medicamentos antidepressivos tricíclicos) por períodos de meses ou anos (Wald, 2020). A terapia empírica deve começar com um suplemento de fibra (Moayyedi et al., 2014). A fibra insolúvel e não fermentável é capaz de acelerar o trânsito intestinal aumentando a biomassa das fezes, levando à estimulação direta da secreção e motilidade e consequentemente ajudando os portadores da SII.

Como opção de primeira linha no controle da dor, utilizam-se os agentes antiespasmódicos, como a mebeverina, brometo de cimetrópio, escopolamina, pinavério etrimebutina. A mebeverina atua na membrana celular da musculatura lisa de maneira seletiva e direta sendo competente no tratamento da hipercontratilidade e 
levando ao relaxamento muscular. Outros medicamentos como brometo de cimetrópio, escopolamina e brometo de pinavério obtiveram resultados semelhantes. A trimebutina é um fármaco agonista dos receptores encefálicos que regulam (receptores $\mu$ e $\delta$ ) ou inibem (receptores $\mathrm{k}$ ) a mobilidade gastrointestinal. Dessa forma, ela auxilia no trânsito intestinal tanto em quadros diarreicos como constipados, sendo inclusive uma escolha terapêutica para crianças (Oświęcimska et al., 2017).

Para os portadores da SII que possuam doenças psiquiátricas associadas, especialmente depressão e ansiedade, é preconizado a utilização de antidepressivos (ADP). Os ADP possuem propriedades analgésicas que não dependem dos efeitos de melhora do humor (Brandt et al., 2009; Ruepert et al., 2011). Os distúrbios na condução nervosa levam à ocorrência de sintomas abdominais resultando em hipersensibilidade a estímulos e uma resposta neuronal hiper-reativa. Em pacientes com SII, ocorrem frequentemente desordens emocionais (transtornos do humor, depressão, raiva, somatização) (Pietrzak et al., 2018). Dessa forma, fica evidente a necessidade do uso dos ADPs. De acordo com essa DFGI, as classes mais utilizadas são os antidepressivos tricíclicos (ADTs) e o inibidores seletivos de receptação de serotonina (ISRS). Uma meta-análise com número de 799 pacientes apontou que antidepressivos podem melhorar significativamente os sintomas globais da SIl quando em doses menores do que as utilizadas para o controle da depressão (RR, 1,38; IC95\%, 1,08-1,77) (Xie et al., 2015). Os ADTs (amitriptilina, desipramina, doxepina, imipramina e trimipramina) são recomendados em sintomas refratários às drogas de primeira escolha, possuindo um alto índice de evidências (Moayyedi et al., 2019). Possuem como mecanismo de ação o bloqueio de receptores muscarínicos (colinérgicos), histaminérgicos, serotonérgicos e mais raramente dopaminérgicos, todavia seu efeito a nível gastrointestinal tende a diminuir o peristaltismo. Além disso, outra classe seria os ISRS (citalopram, fluoxetina e paroxetina), os quais possuem como mecanismo de ação uma suposta analgesia central com bloqueio da transmissão da dor do trato digestivo para o cérebro, além de promover um aumento da velocidade do trânsito intestinal e possuindo propriedades anti-inflamatórias e analgésicas, o que leva a ser uma opção no tratamento da SII-C (Ford et al., 2014; Hookway et al., 2015). É importante destacar que, para a escolha do antidepressivo, levam-se em conta os efeitos adversos dos fármacos e seus benefícios para controle dos subtipos. A eficácia clínica de diferentes tipos de antidepressivo ainda não é totalmente elucidada. Os ADPs provavelmente são mais úteis para SII-D, que é caracterizada por movimentos intestinais induzidos pelo aumento da atividade anticolinérgica (Drossman et al., 2003). Contudo, um estudo realizado apenas com pacientes com SII-D relatou que a amitriptilina resulta em uma redução significativa na incidência de evacuações com consistência amolecida e sensação de tenesmo em comparação com um placebo (Vahedi et al., 2008), o que poderia ser benéfico no controle da SII-D. Valendo do princípio da equipe multidisciplinar, ressalta-se que em momentos de insegurança do profissional na prescrição de tais medicamentos, vale o encaminhamento para um psiquiatra.

Dependendo do quadro, outras linhas de tratamento conhecidas são as dos laxativos e antidiarreicos. Os laxantes osmóticos (polietilenoglicol, sorbitol e hidróxido de magnésio) possuem baixa absorção pelo intestino e induzem secreção de água no lúmen intestinal, contribuindo na passagem das fezes e sendo uma escolha na SII-C (Belsey et al., 2010). Já existem ensaios demonstrando a eficácia de tais drogas, como o polietilenoglicol (PEG) e sua participação na constipação crônica (Oświęcimska et al., 2017). O PEG tem poucos efeitos adversos e possui baixo custo. Secundariamente, pode-se aderir os sequestrantes de ácidos biliares no tratamento. Quanto a SII-D aderimos ao uso de fármacos antidiarreicos como a loperamida, que estimula os receptores pré-sinápticos inibitórios no sistema nervoso entérico com consequente inibição do peristaltismo e da secreção.

Os probióticos auxiliam na manutenção do intestino com flora não patogênica e também podem ser usados como terapia alternativa. No entanto, nenhum estudo demostrou grande benefício, sugerindo que os probióticos não possuem comprovação com base científica, porém não eliminam provável potencial de impacto na SII (Parker et al., 2018; Whelan e Quigley, 2013)

Nos pacientes com SII moderada a grave, e ausência de constipação, os antibióticos podem ser 
recomendados particularmente naqueles com distensão abdominal refratário a outras medicações. O FDA aprovou o rifaximina para o tratamento de SII-D, tendo como mecanismo de ação a ligação irreversível à subunidade beta da RNA polimerase DNA-dependente bacteriana, com consequente inibição da síntese de RNA bacteriano (Pimentel et al., 2011).

\section{Considerações Finais}

A presença dos sintomas psicossomáticos se mostrou em grande parte dos estudos analisados, um fator importante para a instalação ou agravamento da SII. Apesar dos métodos irrefutáveis e necessários já reconhecidos no tratamento da patologia em questão, como mudança no estilo de vida e farmacoterapia, vale valorizar a participação dos profissionais da saúde mental no decorrer da avaliação e tratamento do paciente. O reconhecimento na correlação da SII com os transtornos psiquiátricos, como a depressão e ansiedade, associada ao contexto psicológico atual da sociedade aperfeiçoaria a compreensão e o diagnóstico formal da síndrome em questão, tendo em vista que tais sintomas são capazes de exacerbar o quadro clínico e afetar negativamente os resultados do tratamento, assim como a manutenção e prognóstico da SII (Van Oudenhove et al., 2016). Outra consideração seria a influência direta e positiva em diversas questões públicas e sociais, como a melhora da qualidade de vida do paciente, a diminuição nos gastos públicos da área da saúde e no benefício da relação médico-paciente. Referências

\section{Referências}

Aizawa E, Sato Y, Kochiyama T, Saito N, Izumiyama M, Morishita J. Altered cognitive function of prefrontal cortex during error feedback in patients with irritable bowel syndrome, based on FMRI and dynamic causal modeling. Gastroenterology. 2012; 143:1188-1198. doi: 10.1053/j.gastro.2012.07.104

Baneriee A, Sarkhel S, Sarkar R, Dhali GK. Anxiety and Depression in Irritable Bowel Syndrome. Indian J Psychol Med. 2017; 39(6): 741-745. doi: 10.4103/IJPSYM.IJPSYM_46_17

Belsey JD, Geraint M, Dixon TA. Systematic review and meta analysis: polyethylene glycol in adults with nonorganic constipation. Int J Clin Pract. 2010; 64(7):944-955. doi: 10.1111/j.1742-1241.2010.02397.x.

Borowiec AM, Fedorak RN. The role of probiotics in management of irritable bowel syndrome. Current Gastroenterology Reports. 2007; 9(5): 393-400. doi:10.1007/s11894-007-0048-6

Brandt LJ, Chey WD, Foxx-Orenstein AE, Schiller LR, Schoenfeld PS, Spiegel BM, Talley NJ, Quigley EMM, American College of Gastroenterology Task Force on Irritable Bowel Syndrome. An evidence-based position statement on the management of irritable bowel syndrome. Am J Gastroenterol. 2009; 104(1):S1-S35. doi: 10.1038/ajg.2008.122.

Canavan C, West J, Card T. The epidemiology of irritable bowel syndrome. Clinical Epidemiology. 2014; 6: 71-80 Creed F, Ratcliffe J, Fernandes L, Palmer S, Rigby C, Tomenson B. Outcome in severe irritable bowel syndrome with and without accompanying depressive, panic and neurasthenic disorders. Br J Psychiatry. 2005; 186: 507515. doi: 10.1192/bjp.186.6.507

Coss-Adame E, Rao SS. Brain and gut interactions in irritable

bowel syndrome: new paradigms and new understandings. Current gastroenterology

reports. 2014; 16:379. doi: 10.1007/s11894-014-0379-z

Deary V, Chalder T, Sharpe M. The cognitive behavioural model of medically unexplained symptoms: a theoretical and empirical review. ClinPsychol Rev. 2007;27(7):781-797. doi: 10.1016/j.cpr.2007.07.002 Drossman DA, Hasler WL. Rome IV-Functional GI disorders: Disorders of gut-brain interaction.

Gastroenterology. 2016; 150(6):1257-1261. doi: 10.1053/ j.gastro.2016.03.035

Drossman DA, Toner BB, Whitehead WE, Diamant NE, Dalton CB, Duncan S, Emmott S, Proffitt V, Akman D, Frusciante K, Le T, Meyer K, Bradshaw B, Mikula K, Morris CB, Blackman CJ, Hu Y, Jia H, Li JZ, Koch GG, Bangdiwala SI. Cognitive-behavioral therapy versus education and desipramine versus placebo for moderate to severe functional bowel disorders. Gastroenterol. 2003; 125(1):19-31. doi: 10.1016/s0016-5085(03)00669-3. Farzaneh N, Ghobakhlou M, Moghimi-Dehkordi B, Naderi N, Fadai F. Evaluation of psychological aspects among subtypes of irritable bowel syndrome. Indian J Psychol Med. 2012; 34:144-148. doi: 10.4103/0253- 


\subsection{0}

Flik CE, Laan W, Zuithoff NPA, van Rood YR, Smout AJPM, Weusten BLAM, Whorwell PJ, de Wit NJ Efficacy of individual and group hypnotherapy in irritable bowel syndrome (IMAGINE): a multicentrerandomised controlled trial. Lancet GastroenterolHepatol. 2019; 4(1): 20-31. doi:10.1016/s2468-1253(18)30310-8

Ford AC, Lacy BE, Harris LA, Quigley EM, Moayyedi P. Effect of Antidepressants and Psychological Therapies in Irritable Bowel Syndrome: An Updated Systematic Review and Meta-analysis. Am J Gastroenterol. 2019; 114(1): 21-39. doi:10.1038/s41395-018-0222-5

Ford AC, Moayyedi P, Lacy BE, Lembo AJ, Saito YA, Schiller LR, Soffer EE, Spiegel BMR, Quigley EMM, Task Force on Management of Functional Bowel Disorders. American College of Gastroenterology monograph on the management of irritable bowel syndrome and chronic idiopathic constipation. Am J Gastroenterol. 2014; 109(1):S2-S26. doi: 10.1038/ajg.2014.187

Ford AC, Talley NJ, Schoenfeld PS, Quigley EMM, Moayyedi P. Efficacy of antidepressants and psychological therapies in irritable bowel syndrome: systematic review and meta-analysis. Gut. 2008; 58(3): 367-378. doi:10.1136/gut.2008.163162

Halmos E, Power V, Shepherd S, Gibson PR, Muir JG. A diet low in FODMAPs reduces symptoms of irritable bowel syndrome. Gastroenterol. 2014; 146(1): 67-75. doi: 10.1053/j.gastro.2013.09.046.

Hauser G, Pletikosic S, Tkalcic M. Cognitive behavioral approach to understanding irritable bowel syndrome. World J Gastroenterol. 2014;20(22):6744-6758.doi: 10.3748/wjg.v20.i22.6744.

Hausteiner-Wiehle C, Henningsen P. Irritable bowel syndrome: relations with functional, mental, and somatoform disorders. World J Gastroenterol. 2014; 20(20): 6024-6030. doi: 10.3748/wjg.v20.i20.6024 Hetterich L, Stengel A. Psychotherapeutic Interventions in Irritable Bowel Syndrome. Front. Psychiatry. 2020; 11:286.doi:10.3389/fpsyt.2020.00286

Hookway C, Buckner S, Crosland P, Longson D. Irritable bowel syndrome in adults in primary care: summary of updated NICE guidance. BMJ. 2015; 350: h701. doi: 10.1136/bmj.h701.

Jeffery IB, O'Toole PW, Öhman L, Claesson MJ, Deane J, Quigley EMM, Simrén M. An irritable bowel syndrome subtype defined by species-specific alterations in faecal microbiota. Gut. 2012; 61(7): 997-1006. doi: 10.1136/gutjnl-2011-301501

Johannesson E, Simrén M, Strid H, Bajor A, Sadik R. Physical activity improves symptoms in irritable bowel syndrome: a randomized controlled trial. Am J Gastroenterol. 2011; 106(5): 915-922. DOI:

10.1038/ajg.2010.480

Kassinen A, Krogius-Kurikka L, Mäkivuokko H, Rinttilä T, Paulin L, Corander J, Malinen E, Apajalahti J, Palva A. The fecal microbiota of irritable bowel syndrome patients differs significantly from that of healthy subjects. Gastroenterology. 2007; 133(1):24-33. doi: 10.1053/j.gastro.2007.04.005

Labus J, Gupta A, Gill HK, Posserud I, Mayer M, Raeen H, Bolus R, Simren M, Naliboff BD, Mayer EA. Randomised clinical trial: symptoms of the irritable bowel syndrome are improved by a psycho-education group intervention. Aliment PharmacolTher. 2013; 37(3), 304-315. doi:10.1111/apt.12171

Labus JS, Dinov ID, Jiang Z, Ashe-McNalley C, Zamanyan A, Shi Y. Irritable bowel syndrome in female patients is associated with alterations in structural brain networks. Pain. 2014; 155:137-149. doi:

10.1016/j.pain.2013.09.020

Lacy BE, Mearin F, Chang L, Chey WD, Lembo AJ, Simren M, Spiller R. Bowel Disorders. Gastroenterology. 2016; 150: 1393-1407. doi:10.1053/j.gastro.2016.02.031

Lee C, Doo E, Choi JM, Jang S, Ryu H-S, Lee JY, Oh JH, Park JH, Kim YS, Brain-Gut Axis Research Group of Korean Society of Neurogastroenterology and Motility. J NeurogastroenterolMotil. 2017; 23(3): 349-362. doi:

10.5056/jnm16220

Li L, Xiong L, Zhang S, Yu Q, Chen M. Cognitive-behavioral therapy for irritable bowel syndrome: A metaanalysis. J Psychosom Res. 2014; 77(1), 1-12. doi:10.1016/j.jpsychores.2014.03.006 Liu S, Hagiwara S I, Bhargava A. Early-life adversity, epigenetics, and visceral hypersensitivity. Neurogastroenterology and motility : the official journal of the European Gastrointestinal Motility Society. 2017; 29(9). doi:10.1111/nmo.13170 Longstreth GF, Thompson WG, Chey WD, Houghton LA, Mearin F, Spiller RC. Functional bowel disorders. 
Gastroenterology. 2006; 130(5):1480-1491. doi: 10.1053/j.gastro.2005.11.061

Lovell RM, Ford AC. Global prevalence of and risk factors for irritable bowel syndrome: a meta-analysis. ClinGastroenterolHepatol. 2012; 10(7): 712-721. doi: 10.1016/j.cgh.2012.02.029.

Malinen E, Rinttilä T, Kajander K, Mättö J, Kassinen A, Krogius L, Saarela M, Korpela R, Palva A. Analysis of the fecal microbiota of irritable bowel syndrome patients and healthy controls with real-time PCR. Am J

Gastroenterol. 2005; 100(2): 373-382. doi: 10.1111/j.1572-0241.2005.40312.x.

Moayyedi P, Andrews CN, MacQueen G, Korownyk C, Marsiglio M, Graff L, Kvern B, Lazarescu A, Liu L, Paterson WG, Sidani S, Vanner S. Canadian Association of Gastroenterology Clinical Practice Guideline for the Management of Irritable Bowel Syndrome (IBS). J Can AssocGastroenterol. 2019; 2(1): 6-29. doi: 10.1093/jcag/gwy071

Moayyedi P, Ford AC, Talley NJ, Cremonini F, Foxx-Orenstein AE, Brandt LJ, Quigley EMM. The efficacy of probiotics in the treatment of irritable bowel syndrome: a systematic review. Gut. 2010; 59(3):325-332. doi: 10.1136/gut.2008.167270

Moayyedi P, Quigley EM, Lacy BE, Lembo AJ, Saito YA, Schiller LR, Soffer EE, Spiegel BM, Ford AC. The effect of fiber supplementation on irritable bowel syndrome: a systematic review and meta-analysis. Am Journ Gastroenterolo. 2014; 109(9): 1367-1374.

Muscatello MRA, Bruno A, Pandolfo G, Micò U, Stilo S, Scaffidi M, Consolo P, Tortora A, Pallio S, Giacobbe G, Familiari L, Zoccali R. Depression, anxiety and anger in subtypes of irritable bowel syndrome patients. J ClinPsychol Med Settings. 2010;17(1):64-70. doi: 10.1007/s10880-009-9182-7

O'Mahony SM, Clarke G, Dinan TG, Cryan JF. Irritable Bowel Syndrome and Stress- Related Psychiatric Comorbidities: Focus on Early Life Stress. HandbExpPharmacol. 2017; 239: 219-246. doi: 10.1007/164_2016_128 Oswiecimska J, Szymlak A, Roczniak W, Girczys-Poledniok K, Kwiecien J. New insights into the pathogenesis and treatment of irritable bowel syndrome. Adv in Med Sci. 2017; 62(1): 17-30. doi: 10.1016/j.advms.2016.11.001 Parker EA, Roy T , D'Adamo CR, Wieland LS. Probiotics and gastrointestinal conditions: An overview of evidence from the Cochrane Collaboration. Nutrition. 2018; 45: 125-134.

Pietrzak A, Skrzydlo-Radomanska B, Mulak A, Lipinski M, Malecka-Panas E, Regula J, Rydzewska G. Guidelines on the management of irritable bowel syndrome. In memory of Professor Witold Bartnik. Prz Gastroenterol. 2018; 13(4): 259-288. doi: 10.5114/pg.2018.78343

Pimentel M, Lembo A, Chey WD, Zakko S, Ringel Y, Yu J, Mareya SM, Shaw AL, Bortey E, Forbes WP, TARGET Study Group. Rifaximin therapy for patients with irritable bowel syndrome without constipation. N Engl J Med. 2011; 364(1): 22-32. doi: 10.1056/NEJMoa1004409

Quigley EMM, Fried M, Gwee K-A, Khalif I, Hungin APS, Lindberg G, Abbas Z, Fernandez LB, Bhatia SJ, Schmulson M, Olano C, LeMair A. World Gastroenterology Organisation Global Guidelines Irritable Bowel Syndrome. J ClinGastroenterol. 2016; 50(9): 704-713. doi:10.1097/mcg.0000000000000653

Rajilic-Stojanovic M, Biagi E, Heilig HG, Kajander K, Kekkonen RA, Tims S, de Vos WM. Global and deep molecular analysis of microbiota signatures in fecal samples from patients with irritable bowel syndrome. Gastroenterology. 2011; 141(5):1792-1801. doi: 10.1053/j.gastro.2011.07.043

Ruepert L, Quartero AO, de Wit NJ, van der Heijden GJ, Rubin G, Muris JW. Bulking agents, antispasmodics and antidepressants for the treatment of irritable bowel syndrome. Cochrane Database Syst Ver. 2011; 10(8):CD003460. doi: 10.1002/14651858.CD003460.pub3.

Sayuk GS, Wolf R, Chang L. Comparison of Symptoms, Healthcare Utilization, and Treatment in Diagnosed and Undiagnosed Individuals With Diarrhea-Predominant Irritable Bowel Syndrome. Am J Gastroenterol 2017; 112(6): 892-899.doi: 10.1038/ajg.2016.574

Shah K, Ramos-Garcia M, Bhavsar J, Lehrer P. Mind-body treatments of irritable bowel syndrome symptoms: An updated meta-analysis. Behaviour Research and Therapy. 2020; 128: 103462. doi:10.1016/j.brat.2019.103462 Soares RL. Irritable bowel syndrome: a clinical review. World J Gastroenterol. 2014; 20(34):12144-60. doi: 10.3748/wjg.v20.i34.12144

Spiller R, Aziz Q, Creed F, Emmanuel A, Houghton L, Hungin P, Jones R, Kumar D, Rubin G, Trudgill N, Whorwell $P$, Clinical Services Committee of The British Society of Gastroenterology. Guidelines on the irritable bowel syndrome: mechanisms and practica. Gut. 2007; 56(12): 1770-98. doi: 10.1136/gut.2007.119446 Spiller R. Serotonin and GI clinical disorders. Neuropharmacology. 2008; 55(6): 1072-1080. DOI: 
10.1016/j.neuropharm.2008.07.016

Surdea-Blaga T, Baban A, Nedelcu L, Dumitrascu DL. Psychological Interventions for Irritable Bowel Syndrome. J of Gastrointestin Liver Dis. 2016; 25(3): 359-366. doi: 10.15403/jgld.2014.1121.253.ibs

Tana C, Umesaki Y, Imaoka A, Handa T, Kanazawa M, Fukudo S. Altered profiles of intestinal microbiota and organic acids may be the origin of symptoms in irritable bowel syndrome. Neurogastroenterol Motil. 2010; 22(5):512-519. doi: 10.1111/j.1365-2982.2009.01427.x.

Tanaka Y, Kanazawa M, Fukudo S, Drossman DA. Biopsychosocial model of irritable bowel syndrome. J NeurogastroenterolMotil. 2011; 17(2): 131-9. doi: 10.5056/jnm.2011.17.2.131

Tillisch K, Mayer EA, Labus JS. Quantitative meta-analysis identifies brain regions activated during rectal distension in irritable bowel syndrome. Gastroenterology. 2011; 140:91-100. doi: 10.1053/j.gastro.2010.07.053 Vahedi H, Merat S, Momtahen S, Kazzazi AS, Ghaffari N, Olfati G, Malekzadeh R. Clinical trial: the effect of amitriptyline in patients with diarrhoea-predominant irritable bowel syndrome. Aliment Pharmacol Ther. 2008; 27(8):678-684. doi: 10.1111/j.1365-2036.2008.03633.x.

Van Oudenhove L, Crowell MD, Drossman DA, Halpert AD, Keefer L, Lackner JM, Murphy TB, Naliboff BD, Levy RL. Biopsychosocial aspects of functional gastrointestinal disorders: how central and environmental processes contribute to the development and expression of functional gastrointestinal disorders. Gastroenterology. 2016; 150(6): 1355-1367. doi: 10.1053/j.gastro.2016.02.027

Wald A. Treatment of irritable bowel syndrome in adults. In: Grover S, ed.UpToDate. 2020.Acessado em 29/09/2020 por: https://www.uptodate.com/contents/treatment-of-irritable-bowel-syndrome-in-adults Whelan K, Quigley EM. Probiotics in the management of irritable bowel syndrome and inflammatory bowel disease. Curr Opin Gastroenterol. 2013; 29(2):184-189 doi: 10.1097/MOG.0b013e32835d7bba.

Xie C, Tang Y, Wang Y, Yu T, Wang Y, Jiang L, Lin L. Efficacy and safety of antidepressants for the treatment of irritable bowel syndrome: a meta-analysis. PLoSOne. 2015; 10(8): e0127815. doi:

10.1371/journal.pone.0127815. 